\title{
Alcohol Consumption Prevention Strategies
}

\author{
Lídia Susana Mendes* \\ Nurse with specialization in Health and Psychiatry, University of Lisbon, Portugal \\ *Corresponding author: Lídia Susana Mendes, Nurse with specialization in Health and Psychiatry, University of Lisbon, Portugal. \\ To Cite This Article: Lídia Susana Mendes. Alcohol Consumption Prevention Strategies. Am J Biomed Sci \& Res. 2019 - 3(1). AJBSR.MS.ID.000622. \\ DOI: 10.34297/AJBSR.2019.03.000622
}

Received: May 01, 2019 | Published: May 09, 2019

\section{Opinion/Minireview}

The alcohol consumption affects an increasing number of people around the world, and an estimated 2.3 million over the age of 15 are consuming alcoholic beverages [1]. Binge drinking (BD) is a consumption over 60 gr or more of pure alcohol in one occasion at least one time in the last month, affects young people (15-19 years), with a peak at 20-24 years [1].

There are multiple factors of vulnerability associated with alcohol consumption. The choice of the drink and the age at which the consumptions occur result from the combination of both individual and social vulnerability. The Individual factors that condition alcohol consumption is age, gender, family conditions, religious affiliation, and socioeconomic status (WHO 2014; Collins, 2016). Other conditions, of society, such as the level of development, culture, context of consumption and production of alcohol, distribution and regulation, also have an influence in the consumption of alcoholic beverages.

According to the report of the World Health Organization (2018) the socioeconomic condition is an element to consider in the consumption of alcohol. Alcoholic beverages in many societies are consumed by people of higher socio-economic status since some alcoholic brands are often a symbol of luxury and status. On the other hand, there are alcoholic beverages at reduced prices, accessible to people with low economic income. Hence, this substance, appreciated in elite circles, is accessible to all social classes. However, [2], "damage per liter" has different consequences according to socioeconomic status, the more serious ones occurring in the most disadvantaged classes. Also, the integration of binge drinking into the socialization behaviors of young people and the availability of alcoholic beverages during the 24 hours of the day in several countries have posed enormous challenges in creating effective measures to reduce harmful use of alcohol.

In countries with a long tradition of alcohol consumption, the uncontrolled consumption among young people is unlikely to be prevented merely through repressive measures. Given its cultural dimension, it is essential to learn how to consume alcoholic beverages without causing harm to one's health. For this learning, it will be important to implement interventions in different contexts.
These different contexts that influence consumption refer to the importance of finding partnerships in the community, where the school context is emphasized for a dynamization of actions related with health promotion and prevention of alcohol consumption. Actions should include participation in activities that are felt to be pleasurable, worthy of investment, and which do not include the consumption of alcoholic beverages.

At the level of personal factors, important interventions will be the ones that promote the construction of a health project that includes resources which allow individuals to avoid situations that put their health at risk. The health project should include the resources that each one will use to deal with the life-events that are occurring. Thus, it is important to implement measures that promote the acquisition of knowledge about substances and their consequences, enhance resistance to social pressure, training of personal and social skills, and the construction of safe substance attitudes and expectations [3,4]. Psychoeducational interventions should also include information on the possibility of consumption evolving to dependency, as well as different areas of life that may be affected by the ingestion of alcoholic beverages.

In the community, more specifically in the family and friend's system, each of the participants had the possibility of learning a model of consumption of alcoholic beverages. In the family context, consumptions were allowed, but in reduced doses of alcohol, which, given the relevance of the parental model in learning lifestyles and the acquisition of health behaviors, is an important place where alcohol consumption without risks to health may be learned [5].

\section{Conclusion}

Knowledge about the impact of alcoholic beverages on physical, psychological and social levels is important, so that each one can consciously choose whether or not to integrate it into their habits of life. Using psychoeducational strategies, it is fundamental to make available the information about the impact of this substance on different consumption patterns, and in particular on binge drinking.

\section{References}

1. World Health Organization - WHO (2018) Global status report on alcohol and health. WHO Press, Switzerland. 
2. Katikireddi SV, Whitley E, Lewsey J, Gray L, Leyland AH (2017) Socioeconomic status as an effect modifier of alcohol consumption and harm: analysis of linked cohort data. The Lancet Public health 2(6): e267-e276

3. Barroso T, Mendes A, Barbosa A (2013) Alcohol use / abuse prevention program for adolescents in the school context: stopping to think. Escola Anna Nery 17(3): 466-473.
4. Brito I, Precioso J, Correia C, Albuquerque C, Samorinha C, et al. (2015) Factors associated with alcohol consumption in adolescence, as a function of gender. Psychology, Health \& Diseases 16(3): 392-410.

5. Newman K, Harrison L, Dashiff C, Davies S (2008) Relationships between parenting styles and risk behaviors in adolescent health: an integrative literature review. Rev Lat Am Enfermagem 16(1): 142-150. 